\title{
Valutazione metabolica della nefrolitiasi calcica
}

\author{
M. Marangella, C. Vitale, M. Petrarulo \\ Divisione di Nefrologia e Dialisi e Laboratorio Calcolosi Renale \\ Ospedale Mauriziano Umberto $I^{\circ}$ di Torino
}

impatto sociale della nefrolitiasi calcica è legato alla sua crescente diffusione nelle società occidentali a reddito elevato, con prevalen$\mathrm{ze}$, stimate nella popolazione generale adulta, fra il 4 ed il $10 \%$ (1). La malattia recidiva nel $70-80 \%$ dei casi, contrassegnata dalle sue sequele di colica renale, perdita di giornate di lavoro, necessità di intervento medico ambulatoriale $o$ in ospedale, manovre urologiche chirurgiche o non invasive, e potenziale sviluppo di insufficienza renale. In un recente lavoro abbiamo rilevato fra i pazienti seguiti dal nostro Centro una perdita media di 6.4 giornate lavorative/paziente/anno, e 2.6 giorni di ospedalizzazione paziente/anno (2); circa il $20 \%$ dei pazienti sviluppa una insufficienza renale media-moderata (3). La spesa globale in America per la nefrolitiasi è stata stimata in 1.15 miliardi di dollari nel 1989 (4). Si ritiene che alcune anomalie metaboliche costituiscano fattori causali della nefrolitiasi calcica nella sua forma idiopaticha (5-7). Questa tesi trae origine sia da evidenze sperimentali secondo le quali queste anomalie sono in grado di aumentare il rischio di litogenesi calcica, sia dal riscontro epidemiologico di una incidenza più elevata in soggetti che formano calcoli rispetto a quelli che non ne sono colpiti. Questo ha suggerito una genesi dismetabolica della nefrolitiasi calcica, dando origine ad una gran mole di lavori scientifici negli ultimi venti anni. Alla ipotesi metabolica della nefrolitiasi è sempre stata opposta ed ha ripreso vigore negli ultimi anni l'ipotesi renale secondo la quale il rene sarebbe implicato come protagonista principale nella genesi della calcolosi: anomalie funzionali o anatomiche, primitive o secondarie ad altri eventi patogeni, indurrebbero la formazione, la crescita, l'intrappolamento dei calcoli nel tratto urinario (8-12). Queste ultime ipotesi, sebbene attraenti dal punto di vista speculativo, non hanno ad oggi superato il livello sperimentale e non disponiamo pertanto di procedure attuabili nella routine. Per contro le anomalie metaboliche hanno confermato di avere un significato clinico nella valutazione del rischio di calcolosi $(13,14)$ ed inoltre, grazie alla gran mole di dati accumulati in ampie casistiche, costituiscono ad oggi il mezzo più semplice e riproducibile per il management dei pazienti con nefrolitiasi (15).

Le principali anomalie metaboliche che si ritiene svolgano un ruolo causale e che sono di più frequente riscontro nella nefrolitiasi calcica verranno passate in rassegna in questo lavoro. Verranno proposti alcuni profili diagnostici applicabili nella routine clinica e ne saranno discussi finalità, problemi interpretativi, rapporto costo-benefici.

\section{Indagini diagnostiche}

\section{Esami di laboratorio}

Presso il nostro Centro è in uso un protocollo di indagine ambulatoriale che utilizza sia urine di 24 ore che urine del mattino a digiuno, e campioni di sangue prelevati al termine della raccolta. Al momento della prenotazione i pazienti ricevono istruzioni dettagliate per una corretta raccolta e l'uso dei conservanti (Hibitane, $1 \mathrm{ml}$; $\mathrm{HCl}$ concentrato, $10 \mathrm{ml}$ ). Campioni di sangue ed urine vengono aliquotati da personale tecnico esperto ed inviati a 4 diversi laboratori nell'Ospedale: Calcolosi Renale, Laboratorio Centrale, Ormonologia e Medicina Nucleare. I risultati delle indagini biochimiche ed ormonali vengono implementati ed elaborati per mezzo di un programma computerizzato, che fornisce un referto completo comprendente i parametri misurati e calcolati elencati 
TAB. I - DETERMINAZIONI BIOCHIMICHE E PARAMETRI CALCOLATI NEL PROTOCOLLO DI INDAGINE METABOLICA UTILIZZATO PRESSO IL CENTRO DI CALCOLOSI RENALE DELL'OSPEDALE MAURIZIANO DI TORINO

\section{Determinazioni Biochimiche}

Su Siero e Urine
Urea
Creatinina
Acido Urico
Sodio
Potassio
Cloruri
Calcio
Magnesio
Fosfato

\author{
Escrezioni \\ Acida Netta \\ Azotata Totale
}

\author{
Urine 24 ore \\ Citrato \\ Solfato \\ Ossalato \\ $\mathrm{pH}$ \\ Acidità titolabile \\ Ammonio \\ Bicarbonato
}

\section{Parametri Calcolati}

$$
\begin{gathered}
\text { Clearance } \\
\text { Urea } \\
\text { Creatinina } \\
\text { Acido Urico } \\
\text { Fosfato } \\
\text { Tm Fosfati }
\end{gathered}
$$

\author{
Urine Fasting \\ Creatinina \\ Calcio \\ Citrato \\ Idrossiprolina \\ Ossalato \\ Crosslinks
}

\author{
Altri Marker di Metabolismo Osseo \\ Calcio Ionizzato \\ Fosfatasi Alcalina \\ Paratormone Intatto \\ Osteocalcina \\ Metaboliti della Vitamina D
}

in Tabella I. La maggior parte delle metodiche di dosaggio sono di larga diffusione laboratoristica. Noi utilizziamo la cromatografia ionica per il dosaggio di ossalato, solfato e fosfato urinari $(16,17)$, ma per questi dosaggi sono anche disponibili metodiche non cromatografiche. L'idrossiprolina urinaria viene dosata in HPLC su urine a digiuno ed espressa in mg per grammo di creatinina. Per paratormone, 1 $25(\mathrm{OH})_{2}$ vitamina $\mathrm{D}$, osteocalcina si utilizzano metodiche chemiluminometriche o radioimmunologiche. Un programma dedicato calcola lo stato di saturazione delle urine rispetto ai sali di calcio e di acido urico litogeni (18).

\section{Selezione dei pazienti}

Non tutti i pazienti inviati al nostro Centro vengono sottoposti ad uno screening completo. I criteri di inclusione sono i tabolici completi l'88\% era stato eseguito su pazienti con nefrolitiasi recidivante in fase attiva. Degli altri 116 pazienti $5 \%$ erano in età pediatrica, $23 \%$ donne in età menopausale, $23 \%$ con famigliarità di calcolosi, $25 \%$ con sospetto di malattie sistemiche, ed il restante $24 \%$ miscellanea. L'epoca in cui eseguire la valutazione metabolica è parimenti importante: la presenza di infezione urinaria o di ostruzione può alterare filtrazione glomerulare e funzionalità tubulare. Le abitudini igienico-dietetiche, ed in particolare l'apporto di liquidi, sono spesso diverse dal consueto durante o subito dopo un episodio di calcolosi. Questo può causare una erronea valutazione del rischio litogeno (19) e consiglia di posticipare l'esecuzione degli esami o una loro ripetizione a qualche mese di distanza.

\section{Obiettivi dello screening metabolico}

Le principali finalità dello screening sono qui sotto elencate e discusse.

\section{Accertamento delle anomalie metabo- liche}

L'elevata incidenza di anomalie metaboliche in corso di nefrolitiasi calcica ha giustificato la diffusione di protocolli per la valutazione metabolica dei pazienti. Dai risultati di grosse casistiche in diverse popolazioni è ampiamente accettato che per nessuna di queste anomalie è possibile stabilire una chiara separazione fra normali e litiasici. Questo è naturalmente fonte di controversia nello stabilire i valori di riferimento per ogni parametro. Le principali anomalie metaboliche, i relativi profili diagnostici ed il loro significato clinico saranno discussi sotto. In Tabella II ne è riportata la prevalenza in 1232 pazienti con nefrolitiasi calcica. I valori di riferimento sono relativi a 100 soggetti normali confrontabili per sesso e fasce di età. Nella nostra esperienza iperossaluria ed ipocitraturia sono più frequenti nei pazienti con nefrolitiasi recidivante rispetto a quelli con un solo episodio. Non emerge alcuna differenza rispetto ad altri fattori di rischio.

\section{Definizione dei meccanismi fisiopato- logici}

La comprensione dei meccanismi che causano l'anomalia metabolica costituisce la premessa per una terapia selettiva e mirata. L'applicazione più diffusa dei protocolli diagnostici è quella che mira 
TAB. II - PREVALENZA DELLE PRINCIPALI ANOMALIE METABOLICHE IN 1232 PAZIENTI CON NEFROLITIASI CALCICA RECIDIVANTE E NON, STUDIATI PRESSO IL CENTRO DI CALCOLOSI RENALE DELL'OSPEDALE MAURIZIANO DI TORINO

\begin{tabular}{lccccc}
\hline Parametro & Definizione & \multicolumn{4}{c}{ Prevalenza } \\
& & Totale & $\begin{array}{c}\text { Non } \\
\text { Recidivanti }\end{array}$ & Recidivanti & $p^{*}$ \\
\hline Ipercalciuria & $\mathrm{uCa}>4 \mathrm{mg} / \mathrm{kg} / 24 \mathrm{~h}$ & 39.4 & 44.0 & 36.0 & n.s. 1 \\
Iperossaluria & $\mathrm{uOx}>0.5 \mathrm{mmol} / 24 \mathrm{~h}$ & 17.3 & 6.7 & 21.0 & 0.001 \\
Ipocitraturia & $\mathrm{UCit}<1.5 \mathrm{mmol} / 24 \mathrm{~h}$ & 15.9 & 8.7 & 18.5 & 0.013 \\
Iperuricuria & $\mathrm{uAU}>4 \mathrm{mmol} / 24 \mathrm{~h}$ & 18.5 & 20.0 & 18.0 & n.s. \\
\multicolumn{2}{l}{ Nessuna anomalia } & 31.4 & 33.0 & 30.7 & n.s. \\
\hline
\end{tabular}

*Chi quadro per la differenza fra recidivanti e non recidivanti alla diagnosi differenziale delle ipercalciurie idiopatiche (20). Tuttavia, per questa e per altre anomalie, la stessa difficoltà nel definire gli ambiti di norma si riflette nel tentativo di distinzione in sottoclassi con peculiari meccanismi patogenetici. Un approccio più pratico consiste nel programmare lo studio metabolico in modo da definire la dieta-dipendenza o meno della anomalia in esame, e questo vale sia per l'ipercalciuria che per le altre alterazioni metaboliche (2). In linea generale noi consigliamo una valutazione basale incoraggiando il paziente a non modificare le proprie abitudini dietetiche. I risultati ottenuti su urine di 24 ore vengono confrontati con quelli su urine a digiuno e le differenze vengono utilizzate per stabilire il grado di dietadipendenza. La eventuale raccolta di un diario dietetico nei 2-3 giorni precedenti lo studio fornisce ulteriori informazioni. I risultati dello studio preliminare sono di regola sufficienti per impostare un programma terapeutico oppure per stabilire la necessità di indagini ulteriori, orientate e mirate nei modi di cui si dirà in seguito.

\section{Diagnosi di malattie sistemiche}

Le indagini basali associate a protocolli di indagine orientati, consentono di svelare malattie sistemiche in corso di nefrolitiasi calcica. Nella nostra casistica circa il $20 \%$ dei pazienti con calcolosi calcica erano portatori di anomalie morfo-funzionali renali o di malattie sistemiche. La distribuzione percentuale delle stesse è riportata in Figura 1. Da questa si nota che l'iperparatiroidismo primitivo rende conto della maggior parte di queste anomalie. Seguono il rene a spugna, l'acidosi tubulare distale, le iperossalurie primitive e quella enterica, anomalie delle vie urinarie, sarcoidosi, osteopatie, endocrinopatie varie. Il sospetto diagnostico, per la maggior parte di queste malattie sistemiche o renali, emerge dalla dieta-indipendenza delle alterazioni metaboliche dalle stesse indotte.

\section{Valutazione delle abitudini dietetiche} Le indagini di laboratorio su urine di 24 ore, con i parametri misurati e quelli calcolati, sono una fonte molto preziosa di informazioni sulle abitudini dietetiche dei pazienti, in quanto consentono di confutare o confermare gli esiti del diario dietetico. L'attendibilità di questi dati dipende da alcune premesse essenziali:

a) i pazienti devono essere incoraggiati a non modificare le proprie abitudini nei giorni precedenti la raccolta urinaria;

b) la raccolta deve essere rigorosamente completa e corretta;

c) il paziente deve essere in condizioni stabili e con bilanci metabolici in pareggio.

Per questo lo screening deve essere programmato ad una certa distanza temporale da episodi di colica o calcolo, al fine di evitare il cosiddetto stone-clinic effect (19). Devono essere assegnate istruzioni dettagliate sulle modalità di raccolta urinaria al momento della programmazione. L'anamnesi recente deve escludere qualsiasi causa di potenziale alterazione dello steady-state, quali perdite extra-renali, farmaci o malattie che possano alterare il bilancio esterno di azoto, acido-base ed elettrolitico. Si deve evitare che le indagini metaboliche coincidano con esami strumentali richiedenti una preparazione specifica o diete particolari.

Date queste limitazioni i parametri più importanti sono: escrezione azotata totale - risultante dalla somma dell'azoto di urea, creatinina, acido urico ed ammonio - indice di apporto totale di proteine; escrezione acida netta - somma di ammoniuria ed acidità titolabile meno bicarbonaturia - indice di apporto proteico animale; solfato inorganico ed acido urico, anch'essi marker di proteine animali; sodiuria, indicatore dell'apporto di sale; volume urinario, indicante la compliance alla introduzione di liquidi.

Alcuni parametri possono essere espressi come rapporto con la creatinina urinaria ed agevolmente confrontati con dati analoghi su urine a digiuno. Il confronto dà utili indicazioni sul grado di dieta-dipendenza di una anomalia, e si applica in ge-

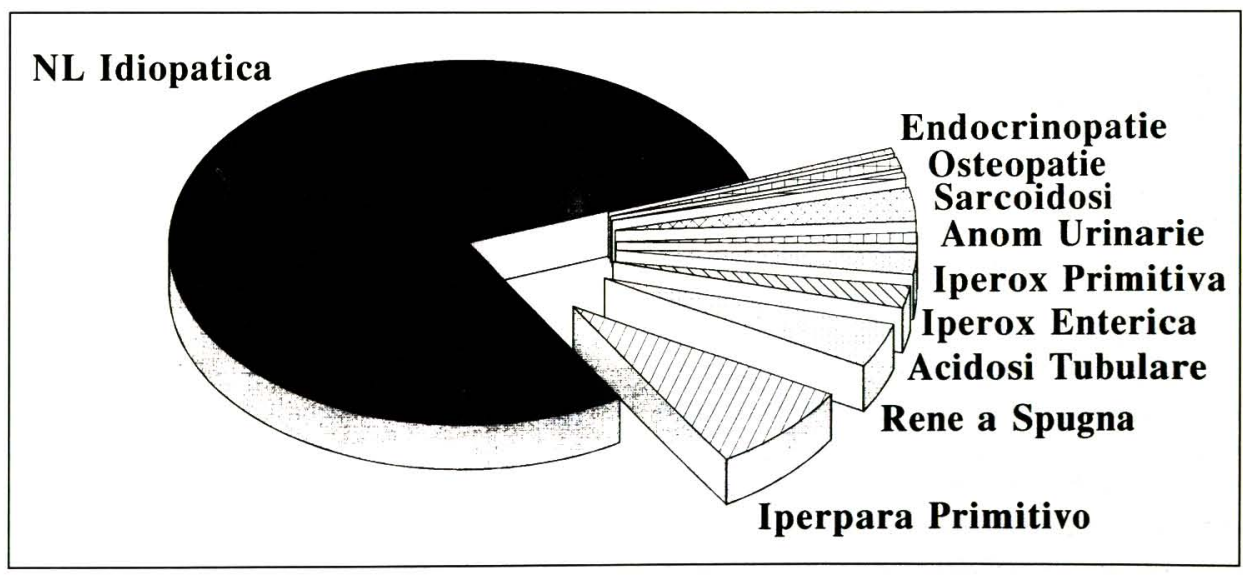

Fig. 1 - Distribuzione percentuale delle cause di nefrolitiasi calcica. 
nere alla escrezione di calcio, citrato, ossalato, acida netta.

\section{Guida al management medico e con-} trollo di compliance

Coe et al in una recente rassegna sulla prevenzione della nefrolitiasi calcica sottolineano che, premessa indispensabile per una terapia efficace, è il riconoscimento dei meccanismi causali della calcolosi (21). Come già accennato, il passo preliminare consiste nel discriminare quei casi in cui la nefrolitiasi è causata da malattie sistemiche oppure da anomalie funzionali o anatomiche renali. In alcune di queste (iperparatiroidismo, acidosi tubulare, iperossalauria enterica, sarcoidosi) la terapia causale spesso consente di determinare una remissione stabile della nefrolitiasi. Nella maggior parte dei casi, quando la nefrolitiasi è definibile idiopatica, l'analisi dei risultati delle indagini biochimiche consente di trarre utili indicazioni su come impostare il management dietetico, correggendo in maniera mirata eventuali eccessi. Di particolare importanza a questo riguardo, sono gli abusi di proteine della carne, sale, zuccheri semplici, prodotti caseari. In alcuni casi il semplice riequilibrio dietetico consente di ottenere una stabile riduzione del rischio litogeno. Noi non favoriamo regimi dietetici troppo restrittivi, né l'uso di diete troppo dettagliate, poiché nel lungo periodo, questo determina una riduzione della aderenza dei pazienti alle prescrizioni. La dieta deve anche tener conto delle complesse interazioni esistenti fra diversi componenti dietetici. Ad esempio, una riduzione troppo drastica di alimenti contenenti calcio aumenta l'assorbimento intestinale e l'escrezione di ossalato, al punto da aumentare paradossalmente il rischio litogeno (22). La riduzione dell'apporto sodico ha due effetti contrastanti: riduce l'escrezione calcica (23), ma riduce anche la solubilità dei sali di calcio in ambiente urinario (24). Una corretta impostazione ed interpretazione delle indagini metaboliche consente di orientarsi nelle scelte da attuare. Nel caso in cui le prescrizioni dietetiche non consentano una efficace correzione del rischio litogeno, si potrà ricorrere ad opzioni farmacologiche, guidate dai risultati di una seconda valutazione metabolica, con il ricorso ai tiazidici, ai supplementi di citrato o di magnesio, ai difosfonati o ad altri farmaci. Infine, la compliance alla terapia pre- scritta potrà essere controllata mediante la programmazione di periodici controlli biochimici, eventualmente mirati.

\section{Valutazione di effetti indesiderati del- la terapia}

Data la sua natura di affezione recidivante e di lunga durata, la prevenzione della nefrolitiasi calcica richiede terapie di lungo periodo. Un problema cruciale nella impostazione del management di questi pazienti è la prevenzione di effetti indesiderati. È noto che restrizioni dietetiche inappropriate e prolungate dell'apporto calcico possono indurre osteopenia $(25,26)$, mentre l'assunzione di tiazidici è stata associata ad una riduzione del rischio di fratture (27). Un effetto negativo sull'assorbimento intestinale e sul bilancio esterno del calcio può essere prodotto dall'uso di fosfato di cellulosa o da diete ricche di fibre (28). Il dosaggio periodico di alcuni marker di riassorbimento osseo, ed il loro confronto con i valori basali, consente di cogliere precocemente alterazioni del metabolismo osseo conseguenti al management medico e di prevenire effetti osteopenizzanti indesiderati. Altri effetti indesiderati indotti dalla dieta o dai farmaci (ipokaliemia, iperuricemia, ipocitraturia, ecc) possono essere svelati ed ovviati dalle indagini biochimiche.

\section{Profili diagnositici delle anomalie metaboliche}

Vengono proposti alcuni protocolli utili alla diagnosi ed alla differenziazione delle anomalie metaboliche che si ritiene svolgano un ruolo causale nella nefrolitiasi calcica.

\section{Ipercalciuria idiopatica}

L'ipercalciuria idiopatica è l'anomalia metabolica di più frequente riscontro nella nefrolitiasi calcica $(2,5,20,29)$. ̀̀ noto che l'escrezione urinaria di calcio ha un pattern di distribuzione continuo, sia nei soggetti normali che nei litiasici (30). Questo è causa di persistenti controversie nella definizione dei cosiddetti intervalli di riferimento per l'escrezione del calcio. La definizione più accettata di ipercalciuria è quella di una escrezione superiore a $4 \mathrm{mg}(0.1 \mathrm{mmol}) / \mathrm{kg} / \mathrm{die}$, oppure $300 \mathrm{mg}(7.5 \mathrm{mmol}) / 24 \mathrm{~h}$ nei maschi, e $250 \mathrm{mg}(6.25 \mathrm{mmol}) / 24 \mathrm{~h}$ nelle femmine. Altra definizione utile è quella del rapporto con la creatinina urinaria, che individua come anomali valori superiori a $0.15 \mathrm{mg}$ di calcio per $\mathrm{mg}$ di creatinina $(0.05 \mathrm{mmol} / \mathrm{mmol})(29)$. Tuttavia, in assenza di malattie sistemiche, l'escrezione calcica dipende ed è modulata da diversi fattori dietetici. Pertanto il concetto di calciuria normale non dovrebbe essere mai disgiunto da quello dell'apporto dietetico, non solo del calcio, ma anche di altri nutrienti quali le proteine, specie quelle di origine animale (31), il sodio (23), zuccheri semplici (31). Pertanto lo studio del calcio urinario dovrebbe essere associato sia ad una accurata inchiesta dietetica, sia alla determinazione di costituenti urinari che sono influenzati e riflettono gli apporti dietetici di alcuni nutrienti. Questo tipo di approccio consente di distinguere le ipercalciurie in dieta-dipendenti e dietaindipendenti. Inoltre, nella interpretazione dei risultati si deve tener conto che $\mathrm{i}$ pazienti con nefrolitiasi calcica a volte rispondono in maniera anomala ad un dato componente della dieta. Analoga considerazione può valere per quanto attiene alla relazione fra intake ed escrezione di calcio, che risente ed è modulata dall'entità dell'assorbimento intestinale (32). In altri termini i meccanismi che regolano il bilancio esterno del calcio possono essere regolati in modo diverso nei litiasici e nei soggetti normali. Tenendo a mente questa impostazione, l'iter diagnostico da adottare nello studio delle ipercalciurie potrebbe essere condotto secondo lo schema illustrato in Figura 2. Questa scheda diagnostica consente di appurare il contributo di singoli componenti dietetici alla escrezione calcica ed offre un utile strumento per la impostazione del trattamento dietetico della ipercalciuria. Inoltre, a partire da questi risultati, quando l'ipercalciuria risulta indipendente dalla dieta, è possibile programmare ulteriori indagini per la diagnosi delle ipercalciurie secondarie o di malattie metaboliche dell'osso (33). Questo si applica a quei pazienti in cui l'escrezione del calcio non si normalizza dopo restrizione dietetica di calcio, proteine e sale, e che presentano ipercalciuria fasting. In questi casi disponiamo oggi di diversi marker di riassorbimento osseo, quali l'idrossiprolinuria dosata in 


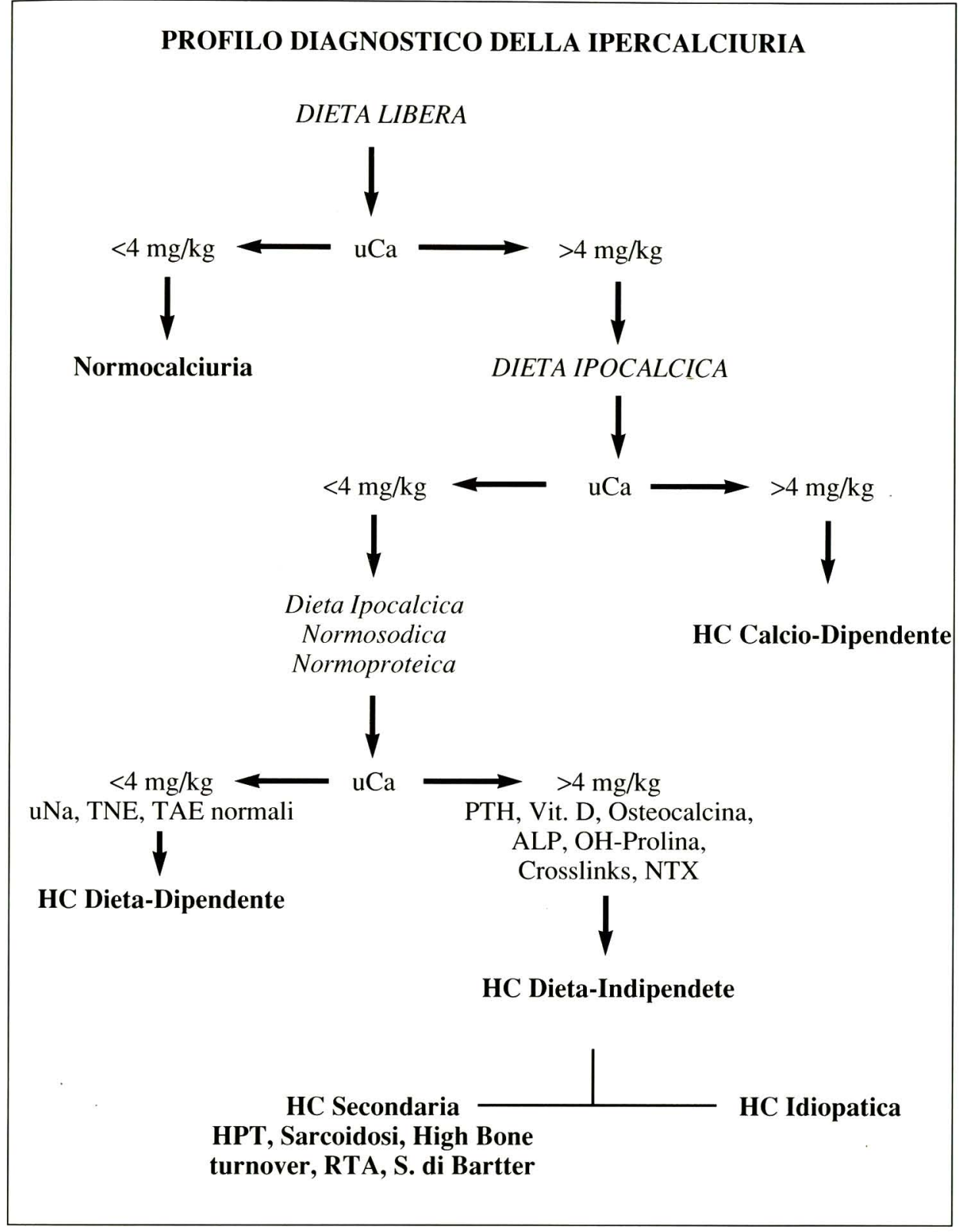

Fig. 2 - Profilo diagnostico della ipercalciuria idiopatica (vedi spiegazione nel testo).

Abbreviazioni: $H C$, ipercalciuria; TNE, escrezione azotata totale; NAE, escrezione acida netta.

HPLC, i crosslinks del piridinio (piridinolina e desossipiridinolina), i frammenti C- o N-terminali del telopeptide del collagene di tipo I, il cui uso è divenuto routinario. La scelta di uno di questi dipende dalle disponibilità operative e dalla strumentazione del laboratorio. Il dosaggio degli ormoni e proteine calcio-regolatori $\left(\mathrm{PTH}\right.$ intatto, $1-25(\mathrm{OH})_{2}$ vitamina $\mathrm{D}$, osteocalcina, isoenzimi della fosfatasi alcalina) è anche potenzialmente utile. Test diagnostici più complessi da applicarsi in caso di ipercalciuria associata a malattie sistemiche comprendono: test da carico con cloruro d'ammonio (acidosi tubulare renale), escrezione frazionale di cloruri, sodio e potassio (sindrome di Bartter), dosaggio del l'ACE o angiotensin converting enzyme (sarcoidosi e malattie granulomatose croniche), soglia renale dei fosfati (sindrome da perdita renale di fosfati).

\section{Iperossaluria mild}

Circa il 20 al $30 \%$ dei pazienti affetti da nefrolitiasi calcio ossalica presenta un aumento della escrezione di ossalato (34-
36). Alcuni Autori negano una significativa incidenza di iperossaluria nella calcolosi idiopatica $(7,13)$, ma riconoscono che l'ossalato ha un ruolo di primo piano quale fattore di rischio di litogenesi calcio ossalica. Il $95 \%$ dei soggetti adulti sani elimina meno di $0.5 \mathrm{mmol}$ ( 45 $\mathrm{mg}) / 24 \mathrm{~h}$ di ossalato, o anche, meno di $50 \mu \mathrm{mol} / \mathrm{mmol}$ di creatinina urinaria (37).

In condizioni normali circa il $90 \%$ dell'ossalato urinario deriva dal metabolismo endogeno: gli acidi gliossilico ed ascorbico ne sono $\mathrm{i}$ più importanti precursori, poiché contribuiscono per il 60$70 \%$, il primo, e per il 10-30\%, il secondo, alla produzione giornaliera di ossalato (38). L'iperossaluria primitiva è caratterizzata da una elevata produzione endogena di ossalato causata da due distinti difetti genetici trasmessi come caratteri recessivi: il tipo 1 (PH1) è dovuto ad un deficit di alanina-gliossilato aminotransferasi epatica e si associa ad iperaciduria glicolica (39). Il tipo 2 ( $\mathrm{PH} 2)$, più raro, è dovuto a deficit D-glicerato-deidrogenasi (gliossilato reduttasi) e si associa ad iperaciduria L-glicerica (40). Secondo alcuni Autori 1'iperossaluria può essere indotta da elevato consumo di proteine di origine animale (41), ma altri Autori negano questo rilievo (42). L'assunzione di dosi farmacologiche di acido ascorbico è un'altra causa controversa di iperossaluria, ammessa da alcuni (43), negata da altri (44). Deficit subclinici di piridossina possono aumentare la produzione endogena di ossalato (45). L'ossalato dietetico contribuisce per meno del $10 \%$ alla generazione giornaliera di questo anione, poiché dei circa 100-200 mg contenuti in una dieta normale, meno del $5-10 \%$ ne vengono assorbiti a livello intestinale (46). Tuttavia, il suo assorbimento intestinale può essere aumentato dalla dieta ipocalcica e da un'elevata assunzione di fibre vegetali (28). L'iperossaluria enterica è una condizione patologica caratterizzata da un iperassorbimento intestinale di ossalato, la quale si associa spesso a sindromi da malassorbimento ed è fondamentalmente causata dalla steatorrea $(38,47)$.

La diagnosi differenziale delle iperossalurie si vale oggi di metodiche affidabili, quali il dosaggio in HPLC di glicolato (48) ed L-glicerato (49), che consentono di diagnosticare le rare forme genetiche. Un aumento della escrezione di glicolato può essere anche causato da deficit di pi- 
ridossina, ed in questo caso può essere differenziato dalla PH1 mediante il dosaggio della transaminasi glutamico ossalacetica intraeritrocitaria (EGOT), ridotta in caso di carenza vitaminica (50). Per contro, glicolato ed L-glicerato urinari sono normali o bassi in caso di carenza vitaminica (50). Per contro, glicolato ed L-glicerato urinari sono normali o bassi in caso di iperossaluria enterica (36). La determinazione dell'acido ascorbico urinario consente di accertare l'abuso di vitamina C come causa di iperossaluria. Tuttavia, dal punto di vista analitico, si deve prestare estrema attenzione alla pesante interferenza dell'ascorbato sulla determinazione dell'ossalato (37).

In Figura 3 è schematizzata una proposta di iter diagnostico per il riconoscimento e la differenziazione delle iperossalurie. Molto controverso è a tutt'oggi il problema della genesi della cosiddetta iperossaluria mild in corso di nefrolitiasi calcica. Escluse le cause note di iperossaluria, quali PH1 e PH2, deficit di piridossina, sindromi da malassorbimento, in un certo numero di pazienti l'iperossaluria rimane inspiegabile. Varie ipotesi sono state fino ad oggi proposte: l'iperossaluria associata ad ipercalciuria assorbitiva si ritiene dovuta ad iperassorbimento intestinale (46). Abbiamo sopra accennato al ruolo controverso attribuito alle proteine animali: la valutazione delle escrezioni azotata totale, acida netta e di solfato inorganico può fornire utili indicazioni nei casi dubbi. Un aumento della clearance renale dell'ossalato $(10,51)$ o lievi anomalie enzimatiche nella biosintesi dell'ossalato (45) sono state anche proposte fra le cause di iperossaluria.

\section{Ipocitraturia idiopatica}

Una riduzione di escrezione e concentrazione del citrato urinario costituisce un fattore di rischio di litogenesi calcica che si realizza attraverso due meccanismi: il citrato è un forte complessante del calcio, e la formazione di complessi calcio citrato riduce la quota di calcio disponibile al legame con l'ossalato (18). In secondo luogo il citrato agisce direttamente come inibitore dei processi di cristallizzazione dei sali di calcio (52).

Il suo handling renale è piuttosto complesso, poiché il citrato può essere utilizzato, dal punto di vista metabolico, dalle cellule tubulari. Pertanto la sua escrezio-

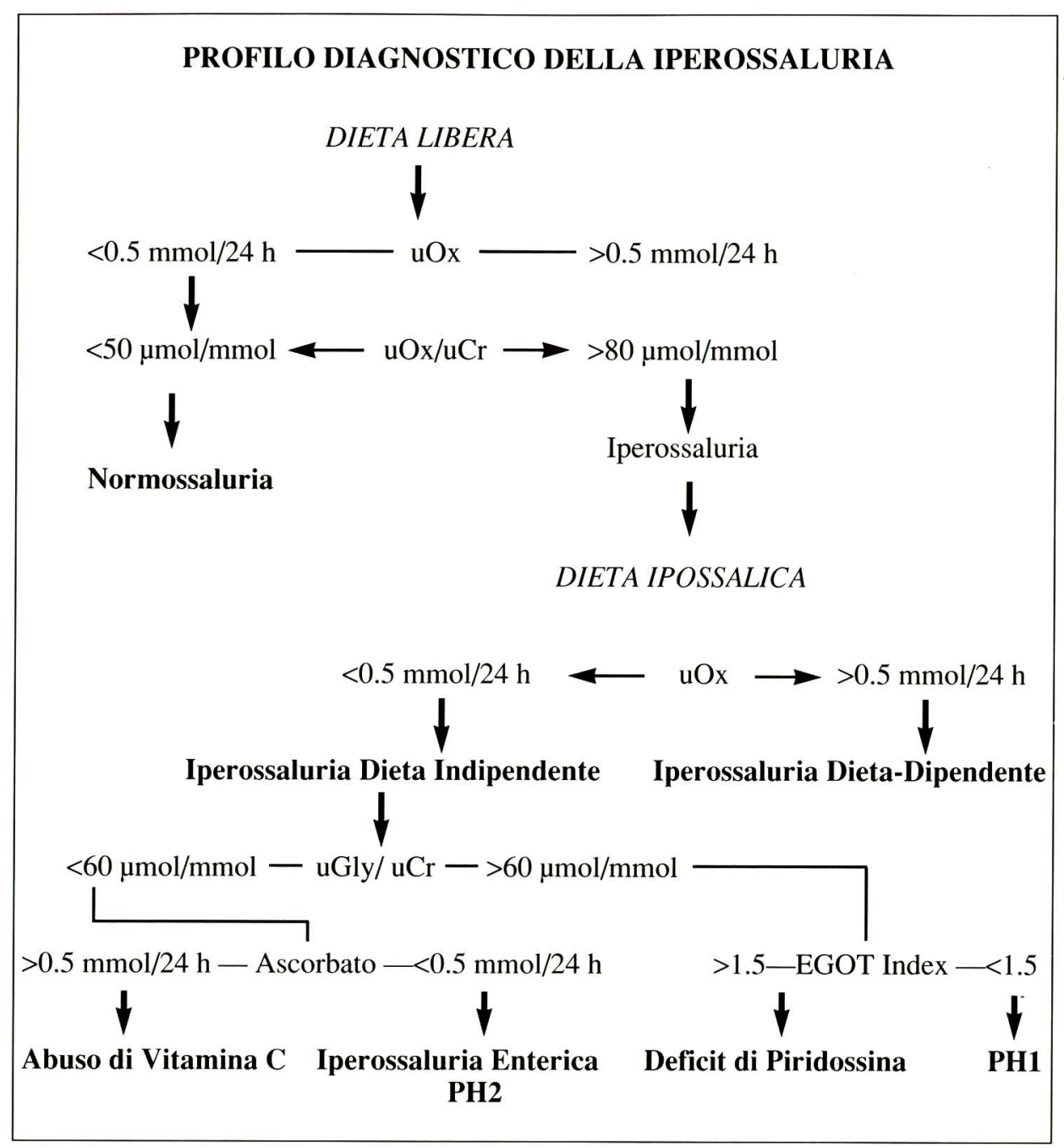

Fig. 3 - Profilo diagnostico della iperossaluria idiopatica (vedi testo). Abbreviazioni: Ox, iperossaluria; EGOT, transaminasi glutamico-ossalacetica eritrocitaria

ne urinaria è il risultato di filtrazione glomerulare, riassorbimento e metabolismo tubulare (53). L'handling renale del citrato è influenzato dall'equilibrio acido-base e dal bilancio elettrolitico, specie quello potassico (53). Inoltre l'insufficienza renale induce importanti modificazioni del suo handling tubulare (54). A causa di queste molteplici interferenze sulla sua escrezione, conviene esprimere i valori di citraturia in rapporto al filtrato glomerulare, e stabilire consensualmente gli intervalli di riferimento. Dal confronto fra 445 pazienti con nefrolitiasi calcica e 100 controlli sani abbiamo definito l'ipocitraturia come una riduzione della escrezione del citrato a valori inferiori a $1.4 \mathrm{mmol}(280 \mathrm{mg}) / 100 \mathrm{ml} \mathrm{di} \mathrm{GFR} / 24 \mathrm{~h}$ (55). Nonostante un'ampia sovrapposi- zione fra normali e litiasici, circa il $17 \%$ dei pazienti con calcolosi calcica possono essere definiti ipocitraturici. L'ipocitraturia può presentarsi isolata, oppure associata ad altre anomalie metaboliche. La Figura 4 mostra uno schema utile per la definizione diagnostca delle ipocitraturie. In via preliminare è utile rapportare la citraturia ai valori di GFR, in modo da differenziare l'ipocitraturia vera dalla riduzione della escrezione del citrato indotta dalla insufficienza renale. La misura della escrezione del citrato anche su urine a digiuno, rapportata alla creatininuria, può fornire una conferma diagnostica, se si tiene conto che vi è una ottima correlazione fra citraturia a digiuno e su urine di 24 h (56). Una eccessiva produzione di acidi fissi, quale quella indot- 


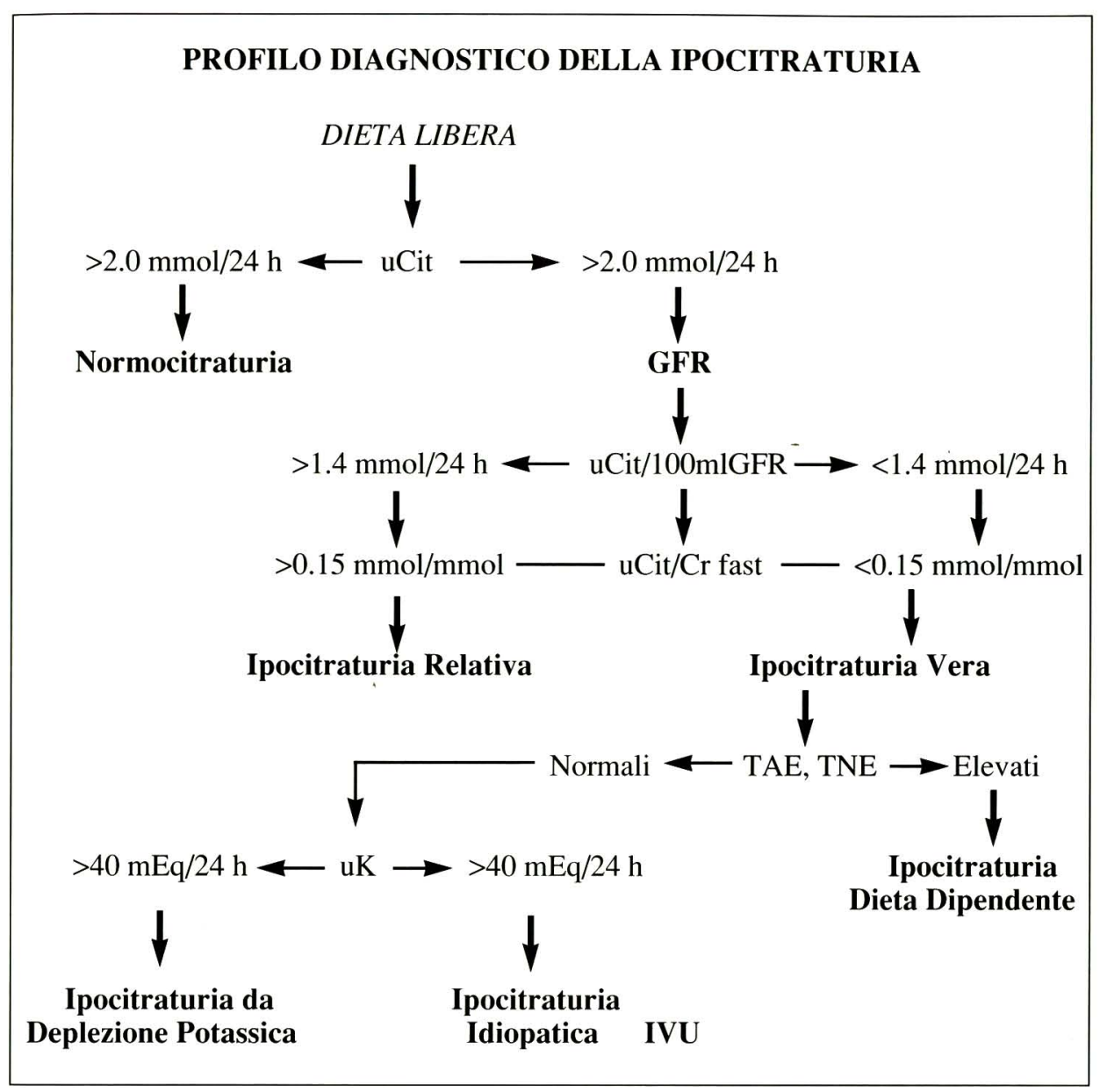

Fig. 4 - Profilo diagnostico della ipocitraturia idiopatica (hCit). Abbreviazioni: hCit, ipocitraturia.

ta da un apporto elevato di aminoacidi solforati, contenuti soprattutto nelle proteine animali, determina una riduzione della citraturia (57), e può essere svelata da un amento di escrezione acida netta e di solfato inorganico. Qualsiasi causa di deplezione potassica induce ipocitraturia $(53,57)$. L'acidosi tubulare renale distale (ATR) induce una riduzione della escrezione di citrato causata da un aumento della sua metabolizzazione renale (53). L'ipocitraturia, associata a ridotta escrezione acida netta deve far porre il sospetto di ATR (58). Le sindromi diarroiche nelle malattie da malassorbimento sono causa di ipocitraturia da deplezione potassica e da perdita di bicarbonati (57): si tenga conto che spesso queste sindromi inducono iperossaluria e che l'ipocitraturia contribuisce in misura cospicua all'aumento del rischio litogeno. si consideri infine che l'infezione urinaria induce una riduzione della escrezione di ci- trato causata da germi capaci di metabolizzare questo anione. E dubbio se in questi casi l'ipocitraturia abbia rilevanza patogenetica per la calcolosi da infezione.

Escluse queste cause di ipocitraturia, in un certo numero di pazienti questa anomalia resta inspiegabile. È stato proposto che nei pazienti con ipocitraturia idiopatica vi possa essere un deficit di assorbimento intestinale di alcali, capace di indurre una modesta e sub-clinica sregolazione del bilancio acido-base (59). Un semplice calcolo consente di stabilire l'assorbimento netto di alcali, e la formula si vale della determinazione di alcuni ioni delle urine: $(\mathrm{Na}+\mathrm{K}+\mathrm{Ca}+\mathrm{Mg})-$ $(\mathrm{Cl}+1.8 \mathrm{P})$, in cui tutti $\mathrm{i}$ valori, salvo quelli del fosforo espressi in $\mathrm{mmol} / 24 \mathrm{~h}$, sono espressi in $\mathrm{mEq} / 24 \mathrm{~h}(60)$. In un nostro studio non pubblicato abbiamo rilevato una incidenza familiare di ipocitraturia e per questo nei casi di persisten- te riduzione del citrato urinario può essere consigliabile estenderne lo studio ai parenti di primo grado.

\section{Iperuricuria}

Il ruolo di una eccessiva escrezione di acido urico nella patogenesi della nefrolitiasi calcica è stato suggerito dalla osservazione che l'incidenza di calcolosi calcica è più elevata nei soggetti gottosi rispetto alla popolazione generale (61), e che una certa quota di pazienti con nefrolitiasi calcica hanno iperuricosuria (62). Inoltre, anomalie dell'handling renale e della escrezione dell'acido urico, isolate $o$ in associazione con ipercalciuria, sono state descritte in pazienti con nefrolitiasi calcica (63). Un sostegno al ruolo dell'urato è stato fornito da dati sperimentali secondo i quali l'urato monosodico favorisce in soluzione la precipitazione del calcio ossalato (64). A queste ipotesi sono state opposte alcune stringenti obiezioni: la cristallogenesi di calcio ossalato avviene in presenza di urato monosodico, ma non di acido urico: le urine di pazienti iperuricurici non sono mediamente più soprasature rispetto all'urato monosodico rispetto a quelle di soggetti normali e di litiasici senza iperuricuria (65). Inoltre l'allopurinolo si è dimostrato inefficace nell'inibire la precipitazione di calcio ossalato nei ratti (66). Infine l'esperienza clinica con l'uso di allopurinolo nella profilassi della nefrolitiasi calcica non ha dato risultati incoraggianti. L'escrezione di acido urico è influenza da un elevato apporto esogeno di purine e questo si associa spesso ad un elevato apporto di proteine animali il cui ruolo patogenetico, come è stato sottolineato sopra, dipende dalla induzione di altre anomalie metaboliche $(31,42$, 59). È dunque verisimile che, al di fuori della calcolosi mista di calcio ed acido urico (67), l'iperuricuria non abbia un rapporto causale con la nefrolitiasi calcica, ma rifletta piuttosto un epifenomeno dovuto ad altri eventi patogenetici.

\section{Stato di saturazione delle urine}

Le anomalie metaboliche, prese singolarmente, possono essere irrilevanti, a meno che non vengano riferite all'ambiente 
urinario nel suo complesso. Le urine debbono essere interpretate come una matrice organica in cui gli ioni più importanti interagiscono fra loro. Da queste molteplici interazioni dipende il rischio litogeno complessivo. A titolo esemplificativo, perfino pazienti con iperossaluria severa possono avere un rischio di litogenesi calcio ossalica relativamente basso in presenza di calciuria bassa e citraturia elevata. In altri termini, non è corretto focalizzare la propria attenzione, dal punto di vista diagnostico e terapeutico, su una singola anomalia metabolica, trascurando gli altri fattori di rischio.

Il calcolo dello stato di saturazione delle urine rispetto ai sali di calcio fosfato e calcio ossalato rappresenta un modello esaustivo, che rende conto delle interazioni ioniche e che misura in modo più complessivo il rischio litogeno.

Ad oggi sono stati proposti due differenti procedure per la stima dello stato di saturazione. La prima utilizza modelli di calcolo computerizzato (ab-initio calculation), in cui lo stato di saturazione risulta dalla soluzione di un sistema di equazioni multiple che tengono conto dei complessi solubili formati fra i principali cationi ed anioni urinari $(14,18)$. La seconda, proposta da Pak, utilizza un metodo semi-empirico, in cui lo stato di saturazione è calcolato dal rapporto fra le concentrazioni ioniche nelle urine originali e quelle misurate dopo incubazione delle stesse per 48 ore con un eccesso del sale in esame (68). Questa tecnica è da ritenersi forse meno complessa, ma più impegnativa e time-consuming.

Noi abbiamo sviluppato un metodo computerizzato, la cui utilizzazione routinaria è stata ormai sperimentata su un gran numero di pazienti $(2,18)$. Il modello consente un calcolo dello stato di saturazione del calcio ossalato $\left(\mathrm{CaOx} . \mathrm{H}_{2} \mathrm{O}\right)$ e della brushite $\left(\mathrm{CaHPO}_{4} .2 \mathrm{H}_{2} \mathrm{O}\right)$, che tiene conto della forza ionica reale delle urine esaminate ed utilizza ben 40 complessi solubili formati fra cationi ed anioni. In pratica, il calcolo richiede che vengano implementati i parametri di laboratorio misurati, elencati in Tabella $\mathbf{I}$. Il programma fornisce i valori di saturazione secondo una scala in cui il valore di 1 indica saturazione, meno di 1 sottosaturazione, superiore ad 1 soprasaturazione. Lo stato di saturazione così espresso fornisce una misura diretta della propensione delle urine alla litogenesi di calcio ossalato e calcio fosfato.
Un limite di questo tipo di approccio è che questo non rende conto del potenziale inibitorio delle urine, una riduzione del quale può avere un'importanza cruciale in molti pazienti $(5,52)$. Tuttavia, il calcolo dello stato di saturazione richiede il dosaggio di citrato e magnesio urinari, due importanti inibitori della cristallizzazione, e questo può fornire indicazioni aggiuntive diagnostico-terapeutiche. Fra gli altri inibitori vi sono i glicosaminoglicani urinari (GAGS) il cui dosaggio routinario è stato proposto da alcuni Autori. A nostro parere, nessuno dei metodi fin qui proposti fornisce risultati abbastanza attendibili. Alcune proteine urinarie hanno una potente azione inibitoria (69). Fra queste, la proteina di Tamm-Horsfall, la nefrocalcina, 1'uropontina, acidi nucleici, ed altre, il cui dosaggio ha spesso carattere sperimentale. Lo stesso dicasi per alcuni metodi semiempirici per la valutazione dell'attività inibitoria delle urine in toto.

\section{Valore predittivo delle indagini metaboliche}

Resta da discutere se lo studio metabolico ha valore predittivo della nefrolitiasi calcica. In teoria, 1 'ipotesi metabolica poggia sull'assunto che vi sia un preciso rapporto fra ambiente urinario e litogenesi. Questo consentirebbe di separare soggetti normali da quelli a rischio di calcolosi, di classificare i litiasici sulla base della presentazione metabolica e di stabilire una relazione fra attività clinica ed entità delle anomalie rilevate. Alcuni Autori hanno studiato modelli predittivi del rischio litogeno. Robertson ha proposto un indice fisico-chimico, rappresentato dal rapporto fra saturazione e potenziale inibitorio delle urine, più elevato fra i litiasici rispetto ai normali $(14,70)$. Parks e Coe trovarono che il rapporto calcio-citrato urinari era più elevato fra $\mathrm{i}$ litiasici (13). Nella nostra esperienza l'i-

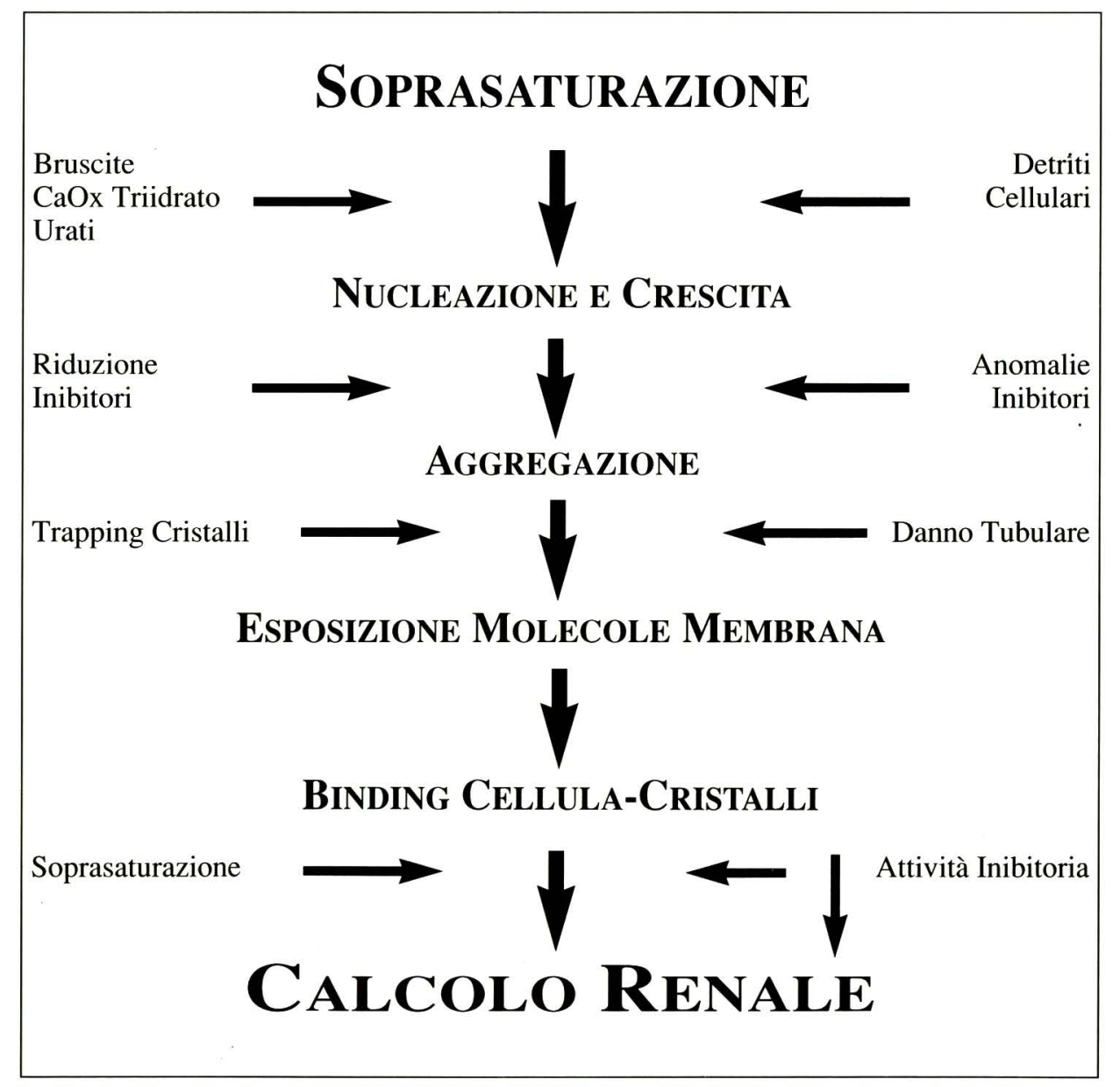

Fig. 5 - Proposta di un modello complessivo di litogenesi calcica. 
perossaluria e l'ipocitraturia sono nettamente più frequenti fra i litiasici recidivanti rispetto ai non recidivanti (2). Tuttavia, nessuno dei modelli suddetti consente una descrizione esauriente del rischio litogeno e della efficacia della profilassi. Molte sorgenti di errore possono alterare il rapporto fra clinica e laboratorio e fra queste, indagini metaboliche eseguite in modo intempestivo, inaccuratezza nella raccolta dei campioni, l'intervento di fattori non rilevabili nell'ambiente urinario.

È pertanto ragionevole assumere che la nefrolitiasi non possa essere spiegata meramente come conseguenza di anomalie metaboliche. I concetti più recenti teorizzano che il rene svolga un ruolo non secondario nella genesi dei calcoli. La riscoperta della teoria di una calcolosi intranefronica suggerisce la necessità di studi che coinvolgano le differenze fra le urine finali e l'urina intra-nefronica, la produzione intra-renale di potenti inibitori della cristallizzazione (71), le interazioni fra cristalli e superfici cellulari ( 72 , 73 ), anomalie primitive del trasporto tubulare di calcio ed ossalato $(12,10)$, danni tubulari pre-esistenti (8). Tuttavia, anche quando si tenga conto di tutti questi nuovi aspetti patogenetici, in una ipotesi complessiva della nefrolitiasi calcica lo studio metabolico dell'ambiente urinario resta un passaggio ineludibile (Fig. 5).

\section{Riassunto}

La nefrolitiasi calcica ha una elevata prevalenza nei paesi sviluppati occidentali. I costi sociali ed economici non indifferenti giustificano gli studi volti a chiarirne le cause. L'elevata incidenza di anomalie metaboliche in corso di nefrolitiasi calcica e la loro azione volta ad aumentare il rischio litogeno è alla base della ipotesi metabolica di questa nefropatia. Diversi protocolli di indagine che si valgono di tecniche di laboratorio moderne e semplificate sono ora disponibili per la valutazione metabolica di questi pazienti. In questo lavoro vengono tracciati e discussi profili diagnostici di ipercalciuria, iperossaluria, ipocitraturia ed iperuricuria, considerandone le possibilità di impiego routinario per lo screening di queste sindromi. Si sottolinea 1'opportunità che il singolo parametro venga considerato come componente dell'ambiente urinario, ove il rischio complessivo di litogenesi deriva dall'interazione dei principali ioni presenti. Questo approccio si prefigge diversi scopi: primo, di pesare il contributo di ciascun componente della dieta e l'eventuale anomala risposta individuale nei confronti di una data anomalia metabolica. Secondo, di separare le calcolosi idiopatiche da quelle secondarie a malattie sistemiche. Terzo di controllare la compliance dei pazienti al trattamento medico e di svelare gli effetti collaterali dello stesso. Le indagini clinico-laboratoristiche di cui si è trattato in questo articolo non costituiscono naturalmente una rassegna completa degli strumenti diagnostici disponibili nel management della nefrolitiasi calcica. Tuttavia, si tratta di metodiche facilmente applicabili nella maggior parte dei centri, riproducibili e di costo relativamente contenuto.

\section{Ringraziamenti}

Il Centro ed il Laboratorio di Calcolosi Renale furono voluti e creati dal prof. Franco Linari, che ci ha ispirato ed incoraggiato nei molti anni di direzione della Divisione di Nefrologia e Dialisi dell'Ospedale Mauriziano di Torino.

Gli Autori ringraziano anche Patrizia Facchini, Eugenio Cerelli e Cristina Mancinetti per la preziosa collaborazione tecnica.

\section{BIBLIOGRAFIA}

1.

Smith LH. The medical aspects of urolithiasis: an overview. J Urol 1989; 141: 707-10.

2. Marangella M. Metabolic evaluation of calcium nephrolithiasis. J Nephrol 1995; 8: 179-84.

3. Marangella M, Bruno M, Cosseddu D, et al. Prevalence of chronic renal insufficiency in the course of idiopathic calcium stone disease: risk factors and patterns of progression. Nephron 1990; 54: 302-6.

4. Pak CYC. Role of medical prevention. J Urol 1989; 141: 789-801.

5. Coe Fl, Favous MJ. Disorders of stone formation. In: Brenner B, Rector FC eds. The Kidney, 3rd ed. Philadelphia: WB Saunders Co. 1986: 1403-42.

6. Broadus AE, Thier SO. Metabolic basis of renal stone disease. $\mathrm{N}$ Engl J Med 1979; 300: 839-43.

7. Pak CYC, Britton F, Peterson $\mathrm{R}$ et al. Ambulatory evaluation of nephrolithiasis: classification, clinical presentation and diagnostic criteria. Am J Med 1980; 69: 19-22.

8. Kumar S, Sigmon DH, Miller T et al. A new model of nephrolithiasis involving tubular dysfunction/injury. J Urol 1991; 146: 1384-9.

9. Ginalski JM, Spiegel TH, Jaeger PH. Does medullary sponge kidney cause nephrolithiasis. Am J Radiol 1990; 155: 299-302.

10. Baggio B, Gambaro G, Marchini F, Cicerello G, Borsatti A. Raised transmembrane flux in red blood cells in idiopathic calcium oxalate nephrolithiasis. Lancet 1984; ii: 11-4.

11. Kok DJ, Khan SR. Calcium oxalate nephrolithiasis, a free or fixed particle disease. Kidney Int 1994; 46: 847-54.

12. Bianchi G, Vezzoli G, Cusi D 
et al. Abnormal red-cell calcium pump in patients with idiopathic hypercalciuria. N Engl J Med 1988; 319: 897-901.

13. Parks JH, Coe FL. A urinary calcium-citrate index for the evaluation of nephrolithiasis. Kidney Int 1986; 30: 85-90.

14. Robertson WG, Peacock M, Nordin BEC. Activity product in stone-forming and non-stone-forming urine. Clin Sci 1968; 34: 579-94.

15. Preminger GM. The metabolic evaluation of patients with recurrent nephrolithiasis: a review of comprehensive and simplified approaches. J Urol 1989; 141: 760-3.

16. Petrarulo M, Bianco O, Martini C, Marangella M, Linari F. Dosaggio degli ioni solfato e fosfato urinari mediante cromatografia ionica. Giorn It Chim Clin 1985; 10: 276-9.

17. Petrarulo M, Marangella M, Bianco O. Preventing ascorbate interference in the ion-chromatographic detrmination of oxalate: four methods compared. Clin Chem 1990; 36: 1642-5.

18. Marangella M, Daniele PG Ronzani $M$ et al. Urine saturation with calcium salts in normal subjects and idiopathic calcium stone formers estimated by an improved computer modelsystem. Urol Res 1985; 13: 189-93.

19. Hosking DH, Erickson SB, Van den Berg CJ, Wilson D, Smith LH. The stone clinic effect in patients with idiopathic calcium urolithiasis. J Urol 1983; 130: 115-9.

20. Pak CYC, Oata M, Lawrence EC, Snyder W. The hypercalciurias: causes, parathyroid functions, and diagnostic criteria. J Clin Invest 1974; 54: 387-400.

21. Coe FL, Parks JH, Asplin JR. The pathogenesis and treatment of kidney stones. N Engl J Med 1992; 327: 1141-52.

22. Smith LH. Diet and hyperoxaluria in syndrome of idiopathic cal- cium urolithiasis. Am J Kidney Dis 1991; 17: 370-5.

23. Muldowney FP, Freaney R, Moloney MF. Importance of dietary sodium in the hypercalciuria syndrome. Kidney Int 1982; 22: 292-6.

24. Daniele PG, Sonego S, Ronzani M, Marangella M. Ionic strength dependence of formation constants. Solubility of calcium oxalate monohydrate and calcium hydrogenphosphate dihydrate in aquaeus solution, at $37^{\circ} \mathrm{C}$ and different ionic strength. Annali Chimica 1985; 75: 245-51.

25. Pietschmann F, Breslau NA, Pak CYC. Reduced vertebral bone density in hypercalciuric nephrolithiasis. J Bone Min Res 1992; 7: 1383-8.

26. Linari F, Marangella M, Vitale C, Cosseddu D. Effetti a lungo termine della terapia medica della nefrolitiasi calcica sul metabolismo minerale. It J Min Elect Met 1992; 6: 89-96.

27. Ray WA, Griffin MR, Downey W, Melton III LJ. Long-term use of phiazide diuretics and risk of hip fracture. Lancet 1989; i: 687-90.

28. Rao P, Jenkins IL, Robertson WG, Peacock M, Blacklock NJ. The effect of high fibre biscuit on urinary risk factors for stone formation. In: Schwille PO, Smith LH, Robertson WG, Vahlensieck W eds. Urolithiasis and related clinical research. New York: Plenum Press, 1985; 425-8.

29. Lemann J Jr, Gray RW. Idiopathic hypercalciuria. J Urol 1989; 141: 715-8.

30. Robertson WG, Morgan DB. The distribution of urinary calcium excretions in normal persons and stone formers. Clin Chim Acta 1972; 37: 503-10.

31. Lemann $\mathbf{J}$ Jr. Urinary calcium excretion and net acid excretion: effects of dietary proteins, carbohydrate and calories. In: Schwille PO, Smith LH, Robertson WG, Vahlensieck W eds. Urolithiasis and related clinical research. New York: Plenum Press, 1985; 53-9.

32. Lemann J Jr, Gray RW. Hypercalciuric kidney stone formers exhibit enhanced intestinal calcium absorption despite only slightly elevated $1,25(\mathrm{OH})_{2}-\mathrm{D}$ concentrations. Kidney Int 1988; 33: 343 (abstract).

33. Sutton RAL, Walker VR. Bone resorption and hypercalciuria in calcium stone formers. Metabolism 1986; 35: 485-8.

34. Robertson WG, Peacock M. The cause of idiopathic calcium stone disease: hypercalciuria or hyperoxaluria? Nephron 1980; 26: 10510.

35. Baggio B, Gambaro G, Favaro S, Borsatti A. Prevalence of hyperoxaluria in idiopathic calcium oxalate kidney stone disease. Nephron 1983; 35: 11-6.

36. Marangella M, Petrrulo M, Vitale C, Cosseddu D, Linari F. Plasma and urine glycolate assays for differentiating the hyperoxaluria syndromes. J Urol 1992; 148: 986-9.

37. Marangella M, Petrarulo M. Oxalate measurement in biological fluids. In: Khan SR ed. Calcium oxalate in biological systems. New York: CRC Press, 1995, cap. 12, pp. 239-63.

38. Williams HE, Wandzilak TR. Oxalate synthesis, transport and the hyperoxalurica syndromes. J Urol 1989; 141: 742-7.

39.

Danpure CJ, Jennings PR, Watts RWE. Enzymological diagnosis of primary hyperoxaluria type 1 by measurement of hepatic alanine: glyoxylate aminotransferase activity. Lancet 1987; i: 289-91.

40. Williams HE, Smith LH Jr. Lglyceric aciduria. A new genetic variant of primary hyperoxaluria. $\mathrm{N}$ Engl J Med 1968; 278: 233-9.

41. Robertson WG, Heyburn PJ, Peacock M, et al. The effect of high animal protein intake on the risk of calcium stone-formation in the urinary tract. Clin Sci 1979; 57: 285-8. 
42. Marangella M, Bianco O, Martini C, Petrarulo M, Vitale C, Linari F. Effect of animal and vegetal protein intake on oxalate excretion in idiopathic calcium stone disease. $\mathrm{Br}$ J Urol 1989; 63: 348-51.

43. Schmidt KH, Hagmaier V, Hornig DH, Vuilleumier JP, Rutishauser $\mathrm{G}$. Urinary oxalate excretion after large intakes of ascorbic acid in man. Am J Clin Nutr 1981; 34: 305-9.

44. Wanszilak TR, D'Andre SD, Davis PA, Williams HE. Effect of high dose vitamine $\mathrm{C}$ on urinary oxalate levels. J Urol 1994; 151 : 834-8.

45. Gill HS, Rose GA. Mild metabolic hyperoxaluria and its response to pyridoxine. Urol Int 1986; 41: 393-6.

46. Marangella M, Bruno M, Fruttero B, Linari F. Hyperoxaluria in idiopathic calcium stone-disease: further evidence of intestinal hyperabsorption of oxalate. Clin Sci 1984; 63: 381-5.

47. Earnest DL, Johnson G, Williams HE, Admirand WH. Hyperoxaluria in patients with ileal resection: an abnormality of dietary oxalate absorption. Gastroenterology 1974; 66: 1114-22.

48. Petrarulo $\mathrm{M}$, Pellegrino $\mathrm{S}$, Bianco O, Marangella M, Linari F, Mentasti E. Derivatization and high performance liquid chromatographic determination of urinary glycolic acid. J Chromatogr 1989; 465: 87-93.

49. Petrarulo M, Marangella M, Cosseddu D, Linari F. High- performance liquid chromatographic assay for L-glyceric acid in body fluids. Application in primary hyperoxaluria type 2. Clin Chim Acta 1992; 211: 143-53.

50. Williams DG. Methods for the estimation of three vitamin dependent red cell enzymes. Clin Biochem 1976; 9: 252-6.

51. Gambaro G, Petrarulo M, Nardelotto A, Marangella M, Baggio B. Erythrocyte transmembrane flux and renal clearance of oxalate in idiopathic calcium nephrolithiasis. Kidney Int 1995; 48: 1549-52.

52. Kok DJ, Papapoulos SE, Bijvoet OLM. Eccessive crystal agglomeration with low citrate excretion in recurrent stone-formers. Lancet 1986; i: 1056-8.

53. Simpson DP. Citrate excretion: a window on renal metabolism. Am J Physiol 1983; 244: F223-34.

54. Marangella M, Vitale C, Manganaro M, et al. Renal handling of citrate in chronic renal insufficiency. Nephron 1991; 57: 439-43.

55. Marangella M, Bianco O, Grande ML et al. Patterns of citrate excretion in healthy subjects and patients with idiopathic stone disease. Contr Nephrol. 58, Karger, Basel 1987; 34-8.

56. Vitale C, Marangella M, Cosseddu D, Bianco O, Linari F. Mechanisms of hypocitraturia in idiopathic calcium stone disease. In: Walker VR, Sutton RAL, Cameron ECB, Pak CYC, Robertson WG eds. Urolithiasis. New York: Plenum Publishing Co. 1989, 501-2.

57. Nicar MJ, Skurla C, Sakhaee $\mathrm{K}$, Pak CYC. Low urinary citrate excretion in nephrolithiasis. Urology 1983; 21: 8-14.

58. Preminger GM, Sakhaee K, Skurla C, Pak CYC. Prevention of recurrent calcium stone formation with potassium citrate therapy in patients with distal renal tubular acidocis. J Urol 1985; 134: 20-3.

59. Pak CYC. Citrate and renal calculi: new insights and future directions. Am J Kidney Dis 1991; 17: 420-5.

60. Oh MS. A new method for estimating GI absorption of alkali. Kidney Int 1989; 36: 915-7.

61. Gutman AB. Uric acid nephrolithiasis. Am J Med 1968; 45: 756-79.

62. Coe FL. Uric acid and calcium oxalate nephrolithiasis. Kidney Int 1983; 24: 392-403.
63. Coe FL, Kavalach AG. Hypercalciuria and hyperuricosuria in patients with calcium nephrolithiasis. N Engl J Med 1974; 291: 1344-50.

64. Pak CYC, Waters O, Arnold LR, Holt K, Cox C, Barilla D. Mechanism of calcium urolithiasis among patients with hyperuricosuria. J Clin Invest 1977; 59: 426-31.

65. Coe FL, Strauss AL, Tembe V, Le Dun S. Uric acid saturation in calcium nephrolithiasis. Kidney Int 1980; 17: 662-8.

66. Finlayson B, Reid F. The effect of allopurinol on calcium oxalate (whewhellite) precipitation. Invest Urol 1978; 15: 489-92.

67. Coe FL. Calcium-uric acid nephrolithiasis. Arch Intern Med 1978; 138: 1090-3.

68. Pak CYC, Holt K. Nucleation and growth of brushite and calcium oxalate in urine of stone formers. Metabolism 1976; 25: 665-73.

69. Kaiser ET, Bock SC. Protein inhibitors of crystal growth. J Urol 1989; 141: 750-2.

70. Robertson WG, Peacock M, Marshall RW, Marshall DH, Nordin BEC. Saturation-inhibition index as a measure of the risk of calcium oxalate stone-formation in the urinary tract. N Engl J Med 1976; 294: 249-52.

71. Coe FL, Nakagawa Y, Parks JH. Inhibitors within the nephron. Am J Kidney Dis 1991; 17: 407-13.

72. Mandel N, Riese R.Crystalcell interactions: crystal binding to rat renal papillary tip collecting duct cells in culture. Am J Kidney Dis 1991; 17: 407-13.

73. Khan SR, Finlayson B, Hackett RL. Experimental calcium oxalate nephrolithiasis, role of renal papilla. Am J Pathol 1982; 107: 5-69. 